\title{
Abnormal Amygdala Resting-State Functional Connectivity in Irritable Bowel Syndrome
}

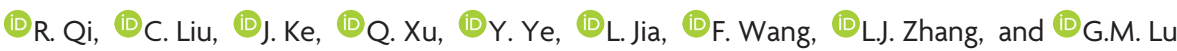

\begin{abstract}
BACKGROUND AND PURPOSE: Functional neuroimaging studies in irritable bowel syndrome have revealed abnormalities in the corticolimbic regions, specifically, hyperactivity of the amygdala during visceral and somatic stimulation. This study investigated changes in the neural circuitry of the amygdala in patients with irritable bowel syndrome based on resting-state functional connectivity.
\end{abstract}

MATERIALS AND METHODS: Functional MR imaging data were acquired from 31 patients with irritable bowel syndrome and 32 healthy controls (matched for age, sex, and educational level) during rest, and the resting-state functional connectivity of bilateral amygdalae was compared. Multiple regression was performed to investigate the relationship between clinical indices of patients with irritable bowel syndrome and resting-state functional connectivity.

RESULTS: Compared with healthy controls, patients with irritable bowel syndrome had higher positive resting-state functional connectivity between the amygdala and insula, midbrain, parahippocampal gyrus, pre- and postcentral gyri, and supplementary motor area. The inclusion of anxiety and depression as covariates did not alter amygdala resting-state functional connectivity differences between the study groups. Multiple covariate regression results showed that the pain intensity in patients with irritable bowel syndrome positively correlated with resting-state functional connectivity between the amygdala and supplementary motor area, pre- and postcentral gyri, and insula, while the Irritable Bowel Syndrome-Symptom Severity Score positively correlated with resting-state functional connectivity between the amygdala and insula and midbrain.

CONCLUSIONS: Patients with irritable bowel syndrome showed disturbed amygdala resting-state functional connectivity with the corticolimbic regions, which could partly account for the enhanced emotional arousal and visceral information processing associated with irritable bowel syndrome.

ABBREVIATIONS: IBS = irritable bowel syndrome; $\mathrm{PHG}=$ parahippocampal gyrus; $\mathrm{RSFC}=$ resting-state functional connectivity; SMA = supplementary motor area

r rritable bowel syndrome (IBS) is a common chronic gastrointestinal condition characterized by abdominal pain, bloating, and disturbed defecation. ${ }^{1,2}$ It is thought to result from a dysregulation of the brain-gut interaction. ${ }^{3}$ With its high prevalence (ob-

Received August 18, 2015; accepted after revision November 16

From the Departments of Medical Imaging (R.Q., J.K., Q.X., L.J.Z., G.M.L.), Gastroenterology (C.L., Y.Y., F.W.), and Emergency Medicine (L.J.), Jinling Hospital, Medical School of Nanjing University, Nanjing, Jiangsu, China.

R.Q., C.L., L.J.Z., and G.M.L. contributed equally to this work.

This work was supported by the grants from the Natural Scientific Foundation of China (Nos. 81322020, 81230032, and 81171313 for L.J.Z. and 81301209 for R.Q.), the Program for New Century Excellent Talents in the University (NCET-12-0260 for L.J.Z.), and the Chinese Key Program (Nos. BWS11J063 and 10z026 for G.M.L.).

Please address correspondence to Long Jiang Zhang, MD, or Guang Ming Lu, MD, Department of Medical Imaging, Jinling Hospital, Medical School of Nanjing University, 305 Zhongshan East Rd, Xuanwu District, Nanjing, Jiangsu Province 210002, China; e-mail: kevinzhlj@163.com; cjr.luguangming@vip.163.com

- Indicates open access to non-subscribers at www.ajnr.org

http://dx.doi.org/10.3174/ajnr.A4655 served in up to $20 \%$ of the population $)^{4}$ and the recurrent nature of the symptoms, IBS negatively affects the quality of life of individuals with this condition and is a health care burden for society ${ }^{5}$; however, the neural basis for IBS has not been examined in detail, to our knowledge.

Functional neuroimaging is the only means of identifying the human brain circuitry that is correlated with various phenotypic and behavioral manifestations of functional gastrointestinal disorders, including $\mathrm{IBS}^{6}$; convergent neuroimaging studies have implicated an abnormal emotional arousal network that encompasses emotional, cognitive, and psychological aspects. ${ }^{7}$ Hyperactivity of the amygdala within this network is a striking feature of IBS, which has been documented in many studies by using visceral or somatic stimuli. ${ }^{6,8}$ The amygdala networks are important in emotional regulation, modulation of sensory information, and processing of visceral information in relation to emotional stimuli. ${ }^{9}$ On the basis of the importance of the amygdala in emotional 
systems and its implication in IBS, this study focused on examining amygdala functional connectivity in patients with IBS.

Brain activity is present even in the absence of an externally activated task, which might cause fluctuations in blood oxygen level-dependent signal. Thus, in task-based activation studies, the background brain activity can distort the blood oxygen leveldependent signal, making it difficult to elucidate the brain mechanisms involved within the studies. Resting-state functional MR imaging is a fairly new approach, which could resolve this issue ${ }^{10}$ by measuring spontaneous blood oxygen level-dependent fluctuations in the brain during rest, ${ }^{11}$ which has been used in the study of IBS. ${ }^{6,12}$ Resting-state functional connectivity (RSFC) - the quantification method most frequently used in resting-state functional MR imaging studies ${ }^{10,13}$ —measures interregional temporal correlation between a predefined seed region and functionally related regions ${ }^{14}$ and has been used as a reliable and sensitive index in studies of healthy subjects and patients with various brain disorders such as Alzheimer disease, depression, and attention deficit/hyperactivity disorder. ${ }^{11,15}$ However, there have been no prior studies using RSFC to investigate the amygdala functional connectivity in patients with IBS.

The present study examined abnormalities of the amygdala functional connectivity in IBS. We hypothesized that amygdala RSFC with corticolimbic regions associated with emotion arousal and visceral information processing would be higher in patients with IBS relative to healthy control subjects, underlying clinically well-observed IBS features such as amplified experience of pain and a negative emotional state. Moreover, given that anxiety and depression have been linked to visceral sensation processing, ${ }^{16}$ we conjectured that anxiety and depression scores would affect amygdala RSFC differences between patients with IBS and healthy controls when included as covariates in the analysis.

\section{MATERIALS AND METHODS Subjects}

All participants provided informed consent to procedures approved by the local Medical Research Ethics Committee. Patients with IBS were recruited from the Digestive Disease Clinic of our hospital and by community advertisements. Thirty-two right-handed patients who were identified as having IBS were recruited for this study between December 2013 and December 2014. A diagnosis of IBS was made by a gastroenterologist with expertise in functional gastrointestinal disorders based on the Rome III criteria, ${ }^{17}$ which include recurrent abdominal pain or discomfort associated with $\geq 2$ of the following: relief/improvement by defecation, onset related to a change in stool frequency, and onset related to a change in stool appearance.

Inclusion criteria for patient recruitment were as follows: older than 18 years of age and a diagnosis positive for IBS based on the Rome III criteria. Exclusion criteria were as follows: a history of gastrointestinal surgery; current or past psychiatric illnesses or substance abuse; treatment with any centrally acting medications such as selective serotonin reuptake inhibitors, aspirin, or nonsteroidal anti-inflammatory drugs for $>2$ weeks before enrollment; major medical or neurologic conditions; and head motion of $>1.0 \mathrm{~mm}$ in translation or $1.0^{\circ}$ in rotation during MR imaging.
One patient with IBS was excluded for excessive head motion. The remaining 31 patients with IBS ( 25 men, 6 women; mean age, $29.23 \pm 9.69$ years) were included in the final analysis.

Thirty-two age-, sex-, and educational level-matched righthanded healthy control subjects ( 25 men, 7 women; mean age, $27.47 \pm 8.64$ years) were recruited by advertisement and were screened through a medical examination for the absence of chronic pain, bowel disturbance, and visceral disease. Other exclusion criteria were the same as those applied to the IBS patient group.

\section{Questionnaires}

Before MR imaging, each subject completed a set of neuropsychological tests, which included the Mini-Mental State Examination, Montreal Cognitive Assessment, and Zung Self-Rating Anxiety and Depression Scales. When making the research plan before the experiment, we included the Mini-Mental State Examination to avoid inclusion of those subjects with possible dementia, ${ }^{18}$ especially for the older subjects who might be recruited, so this test was performed in all subjects, including those with relatively young age. The Montreal Cognitive Assessment ${ }^{19}$ was used to screen subjects for mild cognitive impairment and to evaluate their general cognition, and the Zung Self-Rating Anxiety Scale ${ }^{20}$ and the Zung Self-Rating Depression Scale ${ }^{21}$ were used to rate anxiety-/ depression-related symptoms. In addition, patients with IBS were also tested for the IBS-Symptom Severity Score, ${ }^{22}$ and the IBS-Quality of Life score, ${ }^{23}$ and with the visual analog scale (0-100 points), which included the descriptors "no pain sensation" at zero and "the most intense pain sensation imaginable" at $100 .^{24}$

\section{MR Imaging Data Acquisition}

Subjects were scanned by using a 3T MR imaging scanner (Tim Trio; Siemens, Erlangen, Germany). A foam pad was used to minimize head motion. First, high-resolution T1 structural images were acquired in the sagittal orientation with a magnetization-prepared rapid acquisition of gradient echo sequence (TR/TE, 2300/2.98 ms; flip angle, 9; FOV, $256 \times 256 \mathrm{~mm}^{2}$; acquisition matrix, $256 \times 256$; 191 sections with section thickness of $1 \mathrm{~mm}$ ). Resting-state fMRI data were then obtained by using a single-shot, gradient-recalled echo-planar imaging sequence (250 volumes; TR/TE, 2000/30 ms; FOV, $240 \times 240$ $\mathrm{mm}$; flip angle, $90^{\circ}$; matrix, $64 \times 64$; voxel size, $3.75 \times 3.75 \times$ $4 \mathrm{~mm}$; 30 axial sections aligned along the anterior/posterior commissure).

\section{Data Preprocessing}

Data preprocessing was performed by using SPM8 software (http://www.fil.ion.ucl.ac.uk/spm/software/spm8). For each participant, the first 10 volumes were discarded to account for steady-state longitudinal magnetization and the remaining 240 images were corrected for temporal differences and head motion. Data from 1 patient with IBS were discarded because of excessive head motion. Therefore, 31 patients and 32 controls were included in the analysis. Group differences in translation and rotation of head motion were also evaluated according to the following formula ${ }^{25}$ : 
HeadMotion $/$ Rotation $=$

$$
\frac{1}{L-1} \sum_{i=2}^{L} \sqrt{\left|x_{i}-x_{i-1}\right|^{2}+\left|y_{i}-y_{i-1}\right|^{2}+\left|z_{i}-z_{i-1}\right|^{2}}
$$

where $L$ is the length of the time-series ( $L=240$ in this study), and $x_{i}, y_{i}$, and $z_{i}$ are translations/rotations at the $i$ th time point in the $x$, $y$, and $z$ directions, respectively. No differences in image quality were found between patients with IBS and healthy controls (2sample $t$ test, $t=1.35, P=.18$ for translational motion and $t=$ $1.47, P=.15$ for rotational motion). In this study, T1-weighted images were used as the bridge for the fMRI scans normalized from individual space to the Montreal Neurological Institute space because these T1 structural images had higher resolution than the EPI fMRI scans, which meant that the transformation deviation of individual T1 image to Montreal Neurological Institute space would be smaller than that of the EPI. First, the T1 structural image of each subject was coregistered to the corresponding functional image. Second, the coregistered structural T1 image was segmented by using the unified segmentation algorithm, ${ }^{26,27}$ which can significantly improve the accuracy of spatial normalization, and it was then transformed into the Montreal Neurological Institute space. Third, the functional images were subsequently warped into the standard Montreal Neurological Institute stereotaxic space of $3 \times 3 \times 3 \mathrm{~mm}^{3}$ by applying the parameters of the structural image normalization and then smoothed by convolution with an isotropic Gaussian kernel (8-mm full width at half maximum). After smoothing, imaging data were temporally filtered (bandpass, $0.01-0.08 \mathrm{~Hz}$ ) to remove the effects of low-frequency drift and high-frequency noise. Before functional connectivity analysis, several sources of spurious variance-including 6 head-motion parameters obtained by rigid-body head-motion correction and average signals from CSF and white matter-were removed by applying linear regression. ${ }^{28}$ The global signal was not regressed according to a previous study. ${ }^{29}$

\section{Functional Connectivity Analysis}

The FMRIB Integrated Registration and Segmentation Tool (FIRST; http://fsl.fmrib.ox.ac.uk/fsl/fslwiki/FIRST) was used on T1 images to delineate the amygdalae in each subject; the individual amygdala mask was then normalized to the Montreal Neurological Institute space. For the fMRI data of every subject, the average time-series across all voxels of each amygdala was separately computed as a reference time course and correlated with the time-series of the rest of the brain. Correlation coefficients were then converted to $\mathrm{z}$ values with the Fisher r-to- $\mathrm{z}$ transformation to standardize the statistical analysis. Thus, whole-brain RSFC maps of the bilateral amygdala were generated for each subject.

\section{Statistical Analysis}

SPSS, Version 16.0 (IBM, Armonk, New York) was used to analyze demographic and clinical data. SPM8 was used to analyze RSFC maps for each subject. A random 2-sample $t$ test was then performed to assess differences in amygdala RSFC maps between patients with IBS and healthy controls, while eliminating the effects of age, sex, and educational level by regression. Significant clusters were identified by using the joint expected probability
Table 1: Demographic and clinical data for patients with IBS and healthy controls ${ }^{\mathrm{a}}$

\begin{tabular}{lccc}
\hline \multicolumn{1}{c}{ Protocols } & HC $(\boldsymbol{n}=\mathbf{3 2})$ & Patients $(\boldsymbol{n}=31)$ & $\boldsymbol{P}$ Value \\
\hline Sex (M/F) & $25: 7$ & $25: 6$ & $.81^{\mathrm{b}}$ \\
Mean age (yr) & $27.47 \pm 8.64$ & $29.23 \pm 9.69$ & $.45^{\mathrm{c}}$ \\
Education (yr) & $14.18 \pm 2.33$ & $13.87 \pm 3.24$ & $.66^{\mathrm{c}}$ \\
SAS & $34.44 \pm 5.64$ & $39.74 \pm 9.64$ & $.01^{\mathrm{c}}$ \\
SDS & $36.09 \pm 8.32$ & $41.12 \pm 10.26$ & $.04^{\mathrm{c}}$ \\
MMSE & $27-30 ;$ median, 30 & $28-30 ;$ median, 30 & $.02^{\mathrm{c}}$ \\
MoCA & $28.09 \pm 2.16$ & $27.69 \pm 1.39$ & $.39^{\mathrm{c}}$ \\
IBS-QOL & & $66.05 \pm 12.04$ & \\
IBS-SSS & & $242.48 \pm 28.74$ & \\
VAS (pain) intensity & & $30.47 \pm 14.86$ & \\
Duration (months) & & $32.67 \pm 23.56$ & \\
\hline
\end{tabular}

Note:- $-\mathrm{HC}$ indicates healthy controls; SAS, Self-Rating Anxiety Scale; SDS, Self-Rating Depression Scale; MMSE, Mini-Mental State Examination; MoCA, Montreal Cognitive Assessment; IBS-QOL, IBS-Quality of Life; IBS-SSS, IBS-Symptom Severity Score; VAS, visual analog scale.

${ }^{a}$ Values are expressed as means.

${ }^{\mathrm{b}} P$ value for sex distribution was obtained by the $\chi^{2}$ test.

$c P$ values for age, education, and neuropsychological test scores were obtained by the 2-sample $t$ test.

distribution, ${ }^{29}$ with height $(P<.005)$ and extent $(P<.05)$ thresholds corrected at the whole-brain level.

Statistical analysis was also performed by including anxiety and depression scores (along with age, sex, and educational level) as covariates ${ }^{30}$ to evaluate the effect of anxiety and depression on amygdala RSFC during comparisons. Because the Zung Self-Rating Anxiety Scale and the Self-Rating Depression Scale scores showed high correlations within each group (patient group: Spearman $\rho=0.76, P<.001$; control group: Spearman $\rho=0.78, P<$ .001 ), the scores were taken together (covariates 1 and 2) rather than considered separately as covariates to evaluate the psychosocial effects on the RSFC as described in a previous study of IBS. ${ }^{31}$

A multiple linear regression analysis was used to investigate the relationship between amygdala RSFC in patients with IBS and the IBS-Symptom Severity Score, IBS-Quality of Life score, and pain intensity in SPM8. The statistical threshold was the same as that in the above-mentioned 2-sample $t$ test.

\section{RESULTS}

\section{Clinical Data}

Demographic and clinical information for the subjects is shown in Table 1. There were no differences in terms of age, sex, or educational level between patients with IBS and healthy controls $(P>.05)$. Scores for the Mini-Mental State Examination $(>26)$ and the Montreal Cognitive Assessment $(\geq 26)$ for all subjects were in the normal range, while patients with IBS had lower MiniMental State Examination and higher Zung Self-Rating Anxiety Scale and Depression Scale scores than controls $(P<.05)$ (Table $1)$. All cases were diarrhea-predominant based on bowel habits, with pain reported as a symptom by each patient.

\section{Amygdala Resting-State Functional Network and Effect of Psychosocial Factors}

Patients with IBS had a higher left amygdala positive RSFC with the right insula, midbrain, left pre-/postcentral gyri, right precentral gyrus, left parahippocampal gyrus (PHG), and bilateral supplementary motor areas (SMAs) and higher right amygdala positive RSFC with the right insula, midbrain, left 
PHG, bilateral precentral gyri, and right SMA relative to healthy controls (Table 2 and Figs 1 and 2). No amygdala RSFC differences between patients with IBS and controls were removed when anxiety and depression were included as covariates (along with age, sex, and educational level) in the analysis.

\section{Correlation Analysis}

Multiple covariate regression results suggested that the pain intensity in patients with IBS positively correlated with RSFC be-

\begin{tabular}{|c|c|c|c|c|}
\hline Brain Regions & BA & $\begin{array}{l}\text { MNI Coordinates } \\
(\mathrm{mm})(\mathrm{x}, \mathrm{y}, \mathrm{z})\end{array}$ & $\begin{array}{c}\text { Voxel } \\
\text { No. }\end{array}$ & $\begin{array}{c}T \\
\text { Value }^{\mathrm{a}}\end{array}$ \\
\hline \multicolumn{5}{|l|}{ Left amygdala } \\
\hline Right insula & 13 & $42,6,-3$ & 77 & +3.22 \\
\hline Midbrain & & $12,-30,-21$ & 70 & +3.24 \\
\hline Left pre-/postcentral gyri & 4,9 & $-60,-6,27$ & 78 & +2.89 \\
\hline Right precentral gyrus & 9 & $63,6,27$ & 72 & +2.94 \\
\hline Left PHG & 35 & $-24,-18,-12$ & 77 & +3.31 \\
\hline Left SMA & 6 & $-15,-15,48$ & 98 & +3.18 \\
\hline Right SMA & 6 & $6,-6,60$ & 83 & +3.42 \\
\hline \multicolumn{5}{|l|}{ Right amygdala } \\
\hline Right insula & 13 & $42,12,-12$ & 75 & +3.20 \\
\hline Midbrain & & $12,-30,-21$ & 74 & +3.27 \\
\hline Left PHG & 35 & $-33,-21,-18$ & 76 & +3.57 \\
\hline Left precentral gyrus & 4 & $-53,-8,27$ & 96 & +2.89 \\
\hline Right precentral gyrus & 4 & $63,3,27$ & 80 & +2.94 \\
\hline Right SMA & 6 & $12,-30,60$ & 71 & +3.37 \\
\hline
\end{tabular}

Note:-BA indicates Brodmann area; MNI, Montreal Neurological Institute. ${ }^{a}$ Positive value represents higher positive RSFC. tween the left amygdala and bilateral SMA, pre- and postcentral gyri, and insula; and the RSFC between the right amygdala and the right precentral gyrus and right insula, while the IBS-Symptom Severity Score positively correlated with RSFC between the left amygdala and bilateral insula and the midbrain and with RSFC between the right amygdala and right insula (Fig 3).

\section{DISCUSSION}

The present study investigated changes in amygdala RSFC in IBS. The results showed that patients with IBS had higher RSFC between the amygdala and insula, midbrain, PHG, and sensorimotor regions. In addition, including anxiety and depression as covariates in the analysis had no effect on the RSFC differences between patients with IBS and controls.

\section{Higher Amygdala-Insula RSFC in IBS}

In this study, patients with IBS had higher RSFC between the left and right amygdala and the insula, a major component of the limbic system ${ }^{32}$ that is closely connected to the amygdala. ${ }^{9}$ The insula is involved in emotional arousal and visceral sensory, autonomic visceral motor, and pain processing ${ }^{33}$ and is consistently activated in healthy subjects exposed to visceral stimuli. ${ }^{6}$ The insula is also an essential node in the homeostatic afferent network that is hyperactivated in IBS. ${ }^{8,16,34}$ Increased insula activities in patients with IBS during rectal distension ${ }^{35}$ and in response to cutaneous heat stimuli ${ }^{36}$ have been reported. Evidence also showed that a functional network between the amygdala and insula mediates anxious anticipation of a negative event, and anx-

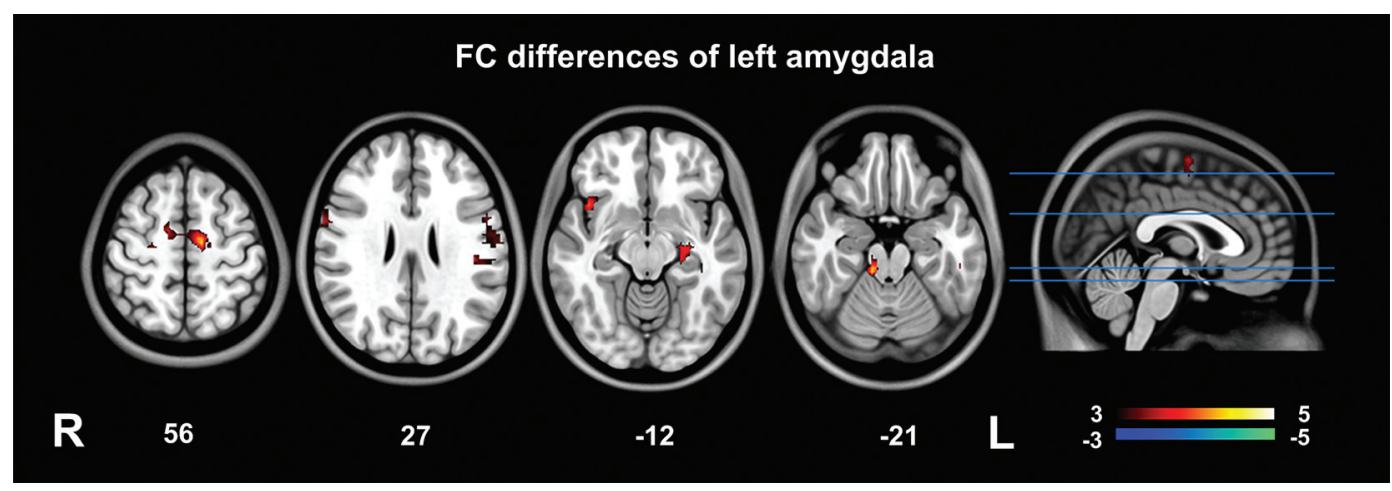

FIG 1. Functional connectivity analysis of the left amygdala. Compared with healthy controls, patients with IBS had higher positive RSFC between the left amygdala and the right insula, midbrain, left pre-/postcentral gyri, right precentral gyrus, left PHG, and bilateral SMA.

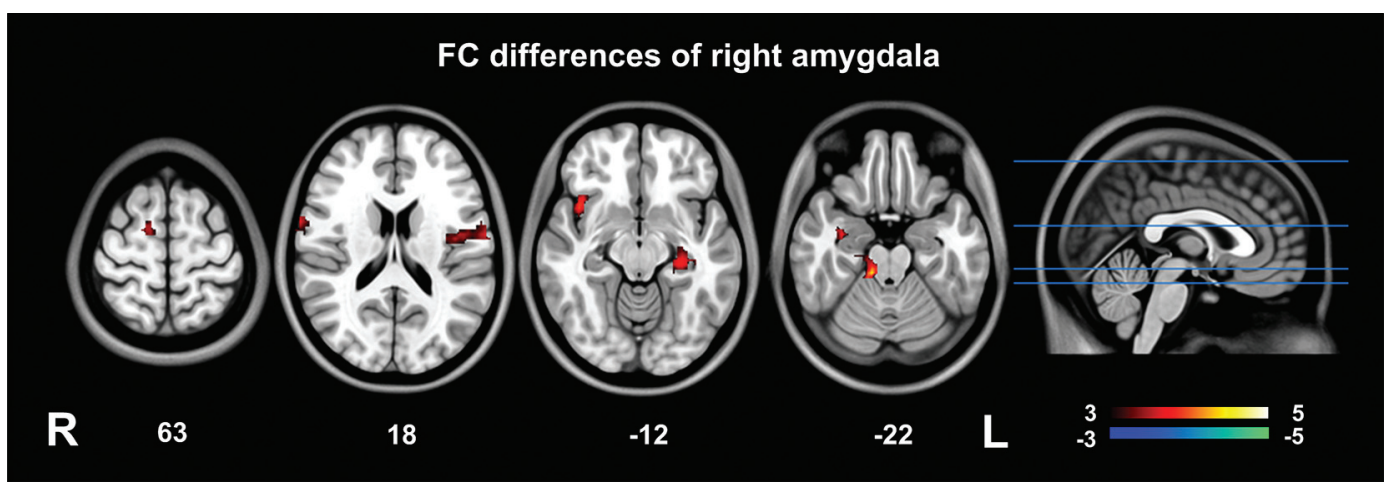

FIG 2. Functional connectivity analysis of the right amygdala. Patients with IBS had higher right amygdala-positive RSFC with the right insula, midbrain, left PHG, bilateral precentral gyri, and right SMA relative to healthy controls. 


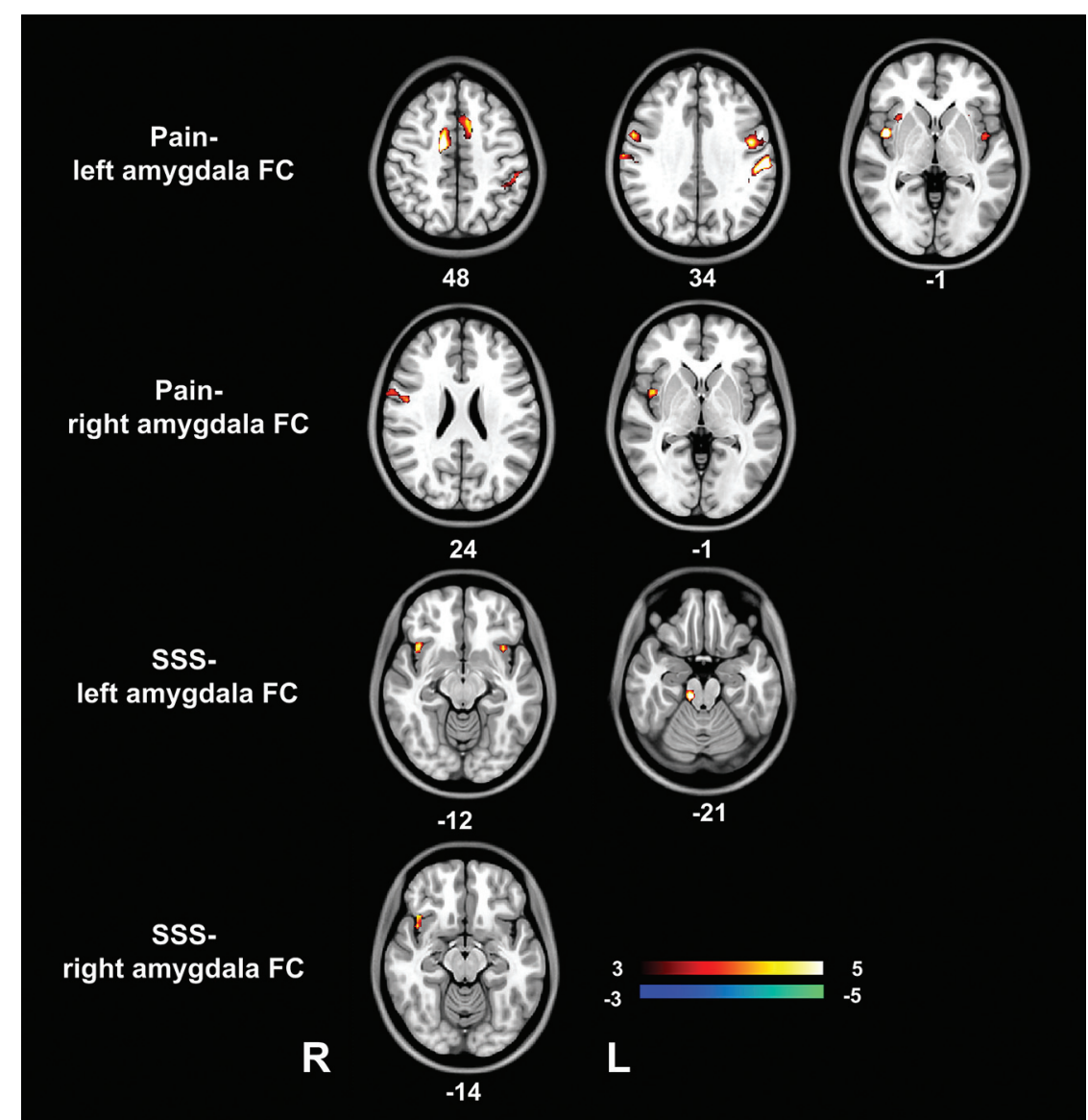

FIG 3. Correlation between amygdala RSFC and clinical indices in patients with IBS. The pain intensity in patients with IBS positively correlated with RSFC between the left amygdala and bilateral SMA, preand postcentral gyri, and insula, and the RSFC between the right amygdala and right precentral gyrus and right insula. The IBS-Symptom Severity Score positively correlated with RSFC between the left amygdala and bilateral insula and midbrain, and RSFC between the right amygdala and right insula. IBS-SSS indicates IBS-Symptom Severity Score; FC, functional connectivity. tween the amygdala and midbrain observed in this study may be related to an amplified nociceptive response to visceral information in IBS. ${ }^{6}$

\section{Higher Amygdala-PHG RSFC in IBS}

The RSFC between the amygdala and PHG was also higher in patients with IBS compared with control subjects. A previous fMRI study of patients with IBS showed that increased hippocampal activity was associated with the anticipation of rectal distension, ${ }^{43}$ and a follow-up study reported a significant correlation between a decreased blood oxygen level-dependent response in the hippocampus during rectal distension and the improvement of gastrointestinal symptoms after the IBS was treated. ${ }^{44}$ The amygdala has extensive connections with the hippocampus and $\mathrm{PHG}^{45}$ which modulate emotional memories. ${ }^{46}$ In addition, a prior fMRI study in healthy adults showed that amygdala-hippocampal connectivity increases during encoding and retrieval of emotional memories. ${ }^{47}$ Thus, in this study, the higher amygdala-PHG RSFC in patients with IBS may arise from long-time encoding of negative emotional memories such as visceral pain. This speculation requires further investigation examining how the amygdala-PHG RSFC changes because of treatment for IBS. ious individuals display exaggerated activity within this network during anticipation of aversive stimuli. ${ }^{37}$ An increase in functional connectivity has been interpreted as increased wiring inside the network or increased neuronal synchrony and/or activity. ${ }^{38,39}$ Thus, the observed higher amygdala-insula functional coupling here may be a mechanism supporting the enhanced emotional arousal and visceral information processing associated with IBS. In addition, the association between insula RSFC and pain intensity in IBS implies a role for the insula in pain processing in these patients. Previous studies have also indicated that the insula is involved in the processing of the affective dimension of pain $^{40}$ and coding of pain intensity. ${ }^{41}$

\section{Higher Amygdala-Midbrain RSFC in IBS}

A higher RSFC between the amygdala and midbrain was detected in IBS relative to control subjects, which has not been previously reported. Previous studies have reported brain stem activation in healthy controls in response to visceral and somatic stimulation ${ }^{42}$ and higher midbrain activation in patients with IBS than in healthy controls during rectal stimulation. ${ }^{8}$ Animal studies have also identified strong connections between the amygdala and brain stem that are responsible for processing visceral information related to emotional stimuli. ${ }^{9}$ We speculate that the higher functional coupling be-

\section{Higher Amygdala-Sensorimotor Region RSFC in IBS}

We also noted a higher RSFC between the amygdala and the sensorimotor regions (SMA, pre- and postcentral gyri). The SMA plays an important role in motor inhibition and response ${ }^{48}$ and the functional processing of painful stimuli. ${ }^{49}$ Pre- and postcentral gyri are important components of motor and sensory areas. These sensorimotor regions, which are core regions within the so-called pain network, ${ }^{40}$ are commonly observed with enhanced activations in IBS and other functional gastrointestinal disorders. ${ }^{50}$ Using resting-state functional MR imaging, Cifre et al ${ }^{39}$ reported increased connectivity between the primary motor cortex and SMA in patients with fibromyalgia, another chronic pain condition, and interpreted that as the result of the widespread central sensitization in patients. Thus, we speculated that the higher RSFC in this study may be related to the central pain amplification in patients with IBS. ${ }^{8}$

\section{Psychosocial Effects on the Amygdala RSFC in IBS}

In this study, anxiety and depression had no effect on amygdala RSFC differences between patients with IBS and healthy controls, contrary to our hypothesis that RSFC abnormalities in IBS could be partly explained by anxiety and depression symptoms. The 
negative finding may be due to the small number of patients with IBS with severe anxiety or depression symptoms that was included in this study and requires confirmation in a larger sample of patients with IBS.

\section{Limitations}

This study had some limitations. First, the study population was relatively small and consisted of Chinese patients with diarrheapredominant IBS; thus, the results may not be generalizable to patients of other ethnicities or with other subtypes of IBS. Second, the exact neurophysiologic meaning of functional connectivity still remains unclear, and much work is needed before it can be used routinely in the clinical setting. Longitudinal studies are also needed to address whether the observed results are altered by IBS treatment. Third, sex differences in IBS $^{51}$ were not considered due to the small number of female patients. Sex differences in brain responses to emotion-related stimuli ${ }^{52}$ and in the resting state $e^{53}$ have been observed in patients with IBS; thus, the potential role of sex in amygdala RSFC should be addressed in future studies. Fourth, although we used the whole amygdala as a seed region, previous research has shown that amygdala subregions have distinct functional networks, ${ }^{54}$ and future investigations should consider variations in RSFC pattern across these subregions.

\section{CONCLUSIONS}

Patients with IBS had abnormalities in amygdala RSFC in several corticolimbic regions, which could, in part, underlie the enhanced emotional arousal and visceral information processing associated with IBS.

Disclosures: Long Jiang Zhang—RELATED: Grant: Natural Scientific Foundation of China; the Program for New Century Excellent Talents in the University, ${ }^{*}$ Comments: grant Nos. 81322020, 81230032, and 81171313; the Program for New Century Excellent Talents in the University (NCET-12-0260). *Money paid to the institution.

\section{REFERENCES}

1. Chey WD, Kurlander J, Eswaran S. Irritable bowel syndrome: a clinical review. JAMA 2015;313:949-58 CrossRef Medline

2. Mayer EA. Clinical practice: irritable bowel syndrome. $N$ Engl J Med 2008;358:1692-99 CrossRef Medline

3. Mayer EA, Tillisch $\mathrm{K}$. The brain-gut axis in abdominal pain syndromes. Annu Rev Med 2011;62:381-96 CrossRef Medline

4. Longstreth GF, Wolde-Tsadik G. Irritable bowel-type symptoms in HMO examinees: prevalence, demographics, and clinical correlates. Dig Dis Sci 1993;38:1581-89 CrossRef Medline

5. Canavan C, West J, Card T. Review article: the economic impact of the irritable bowel syndrome. Aliment Pharmacol Ther 2014;40: 1023-34 CrossRef Medline

6. Mayer EA, Aziz Q, Coen S, et al. Brain imaging approaches to the study of functional GI disorders: a Rome working team report. $\mathrm{Neu}$ rogastroenterol Motil 2009;21:579-96 CrossRef Medline

7. Al Omran Y, Aziz Q. Functional brain imaging in gastroenterology: to new beginnings. Nat Rev Gastroenterol Hepatol 2014;11:565-76 CrossRef Medline

8. Tillisch K, Mayer EA, Labus JS. Quantitative meta-analysis identifies brain regions activated during rectal distension in irritable bowel syndrome. Gastroenterology 2011;140:91-100 CrossRef Medline

9. Price JL. Comparative aspects of amygdala connectivity. Ann N Y Acad Sci 2003;985:50-58 Medline

10. Fox MD, Raichle ME. Spontaneous fluctuations in brain activity observed with functional magnetic resonance imaging. Nat Rev Neurosci 2007;8:700-11 CrossRef Medline

11. Lee MH, Smyser CD, Shimony JS. Resting-state fMRI: a review of methods and clinical applications. AJNR Am J Neuroradiol 2013;34: 1866-72 CrossRef Medline

12. Rapps N, van Oudenhove L, Enck P, et al. Brain imaging of visceral functions in healthy volunteers and IBS patients. J Psychosom Res 2008;64:599-604 CrossRef Medline

13. Fox MD, Greicius M. Clinical applications of resting-state functional connectivity. Front Syst Neurosci 2010;4:19 CrossRef Medline

14. van de Ven VG, Formisano E, Prvulovic D, et al. Functional connectivity as revealed by spatial independent component analysis of fMRI measurements during rest. Hum Brain Mapp 2004;22:165-78 CrossRef Medline

15. Li F, He N, Li Y, et al. Intrinsic brain abnormalities in attention deficit hyperactivity disorder: a resting-state functional MR imaging study. Radiology 2014;272:514-23 CrossRef Medline

16. Van Oudenhove L. Visceral sensory and cognitive-affective neuroscience: towards integration? Gut 2010;59:431-32 CrossRef Medline

17. Drossman DA. The functional gastrointestinal disorders and the Rome III process. Gastroenterology 2006;130:1377-90 CrossRef Medline

18. Folstein MF, Robins LN, Helzer JE. The Mini-Mental State Examination. Arch Gen Psychiatry 1983;40:812 CrossRef Medline

19. Nasreddine ZS, Phillips NA, Bédirian V, et al. The Montreal Cognitive Assessment, MoCA: a brief screening tool for mild cognitive impairment. J Am Geriatr Soc 2005;53:695-99 CrossRef Medline

20. Zung WW. A rating instrument for anxiety disorders. Psychosomatics 1971;12:371-79 CrossRef Medline

21. Zung WW, Richards CB, Short MJ. Self-rating depression scale in an outpatient clinic: further validation of the SDS. Arch Gen Psychiatry 1965;13:508-15 CrossRef Medline

22. Francis CY, Morris J, Whorwell PJ. The irritable bowel severity scoring system: a simple method of monitoring irritable bowel syndrome and its progress. Aliment Pharmacol Ther 1997;11:395-402 CrossRef Medline

23. Patrick DL, Drossman DA, Frederick IO, et al. Quality of life in persons with irritable bowel syndrome: development and validation of a new measure. Dig Dis Sci 1998;43:400-11 CrossRef Medline

24. Price DD, Bush FM, Long S, et al. A comparison of pain measurement characteristics of mechanical visual analogue and simple numerical rating scales. Pain 1994;56:217-26 CrossRef Medline

25. Liao W, Chen H, Feng $Y$, et al. Selective aberrant functional connectivity of resting state networks in social anxiety disorder. Neuroimage 2010;52:1549-58 CrossRef Medline

26. Ashburner J, Friston KJ. Unified segmentation. Neuroimage 2005;26: 839-51 CrossRef Medline

27. Liu CH, Li F, Li SF, et al. Abnormal baseline brain activity in bipolar depression: a resting state functional magnetic resonance imaging study. Psychiatry Res 2012;203:175-79 CrossRef Medline

28. Fox MD, Snyder AZ, Vincent JL, et al. The human brain is intrinsically organized into dynamic, anticorrelated functional networks. Proc Natl Acad Sci U S A 2005;102:9673-78 CrossRef Medline

29. Saad ZS, Gotts SJ, Murphy K, et al. Trouble at rest: how correlation patterns and group differences become distorted after global signal regression. Brain Connect 2012;2:25-32 CrossRef Medline

30. Poline JB, Worsley KJ, Evans AC, et al. Combining spatial extent and peak intensity to test for activations in functional imaging. Neuroimage 1997;5:83-96 CrossRef Medline

31. Zhou G, Qin W, Zeng F, et al. White-matter microstructural changes in functional dyspepsia: a diffusion tensor imaging study. Am J Gastroenterol 2013;108:260-69 CrossRef Medline

32. Augustine JR. Circuitry and functional aspects of the insular lobe in primates including humans. Brain Res Brain Res Rev 1996;22: 229-44 CrossRef Medline

33. Nagai M, Kishi K, Kato S. Insular cortex and neuropsychiatric 
disorders: a review of recent literature. Eur Psychiatry 2007;22: 387-94 CrossRef Medline

34. Chang $\mathrm{L}$. Brain responses to visceral and somatic stimuli in irritable bowel syndrome: a central nervous system disorder? Gastroenterol Clin North Am 2005;34:271-79 CrossRef Medline

35. Yuan YZ, Tao RJ, Xu B, et al. Functional brain imaging in irritable bowel syndrome with rectal balloon-distention by using fMRI. World J Gastroenterol 2003;9:1356-60 CrossRef Medline

36. Verne GN, Himes NC, Robinson ME, et al. Central representation of visceral and cutaneous hypersensitivity in the irritable bowel syndrome. Pain 2003;103:99-110 CrossRef Medline

37. Carlson JM, Greenberg T, Rubin D, et al. Feeling anxious: anticipatory amygdalo-insular response predicts the feeling of anxious anticipation. Soc Cogn Affect Neurosci 2011;6:74-81 CrossRef Medline

38. Bettus G, Guedj E, Joyeux F, et al. Decreased basal fMRI functional connectivity in epileptogenic networks and contralateral compensatory mechanisms. Hum Brain Mapp 2009;30:1580-91 CrossRef Medline

39. Cifre I, Sitges C, Fraiman D, et al. Disrupted functional connectivity of the pain network in fibromyalgia. Psychosom Med 2012;74:55-62 CrossRef Medline

40. Price DD. Psychological and neural mechanisms of the affective dimension of pain. Science 2000;288:1769-72 CrossRef Medline

41. Derbyshire SW, Jones AK, Gyulai F, et al. Pain processing during three levels of noxious stimulation produces differential patterns of central activity. Pain 1997;73:431-45 CrossRef Medline

42. Dunckley P, Wise RG, Fairhurst M, et al. A comparison of visceral and somatic pain processing in the human brainstem using functional magnetic resonance imaging. J Neurosci 2005;25:7333-41 CrossRef Medline

43. Larsson $\mathrm{MB}$, Tillisch $\mathrm{K}$, Craig $\mathrm{AD}$, et al. Brain responses to visceral stimuli reflect visceral sensitivity thresholds in patients with irritable bowel syndrome. Gastroenterology 2012;142:463-472.e3 CrossRef Medline

44. Lowén MB, Mayer EA, Sjöberg M, et al. Effect of hypnotherapy and educational intervention on brain response to visceral stimulus in the irritable bowel syndrome. Aliment Pharmacol Ther 2013;37: 1184-97 CrossRef Medline

45. Hamann SB, Ely TD, Grafton ST, et al. Amygdala activity related to enhanced memory for pleasant and aversive stimuli. Nat Neurosci 1999;2:289-93 CrossRef Medline

46. Izquierdo I, Medina JH. Memory formation: the sequence of biochemical events in the hippocampus and its connection to activity in other brain structures. Neurobiol Learn Mem 1997;68:285-316 CrossRef Medline

47. Smith AP, Stephan KE, Rugg MD, et al. Task and content modulate amygdala-hippocampal connectivity in emotional retrieval. Neuron 2006;49:631-38 CrossRef Medline

48. Nachev P, Kennard C, Husain M. Functional role of the supplementary and pre-supplementary motor areas. Nat Revi Neurosci 2008;9: 856-69 CrossRef Medline

49. Craggs JG, Price DD, Verne GN, et al. Functional brain interactions that serve cognitive-affective processing during pain and placebo analgesia. Neuroimage 2007;38:720-29 CrossRef Medline

50. Jones MP, Dilley JB, Drossman D, et al. Brain-gut connections in functional GI disorders: anatomic and physiologic relationships. Neurogastroenterol Motil 2006;18:91-103 CrossRef Medline

51. Chang L, Heitkemper MM. Gender differences in irritable bowel syndrome. Gastroenterology 2002;123:1686-701 CrossRef Medline

52. Labus JS, Gupta A, Coveleskie K, et al. Sex differences in emotionrelated cognitive processes in irritable bowel syndrome and healthy control subjects. Pain 2013;154:2088-99 CrossRef Medline

53. Hong J-Y, Kilpatrick LA, Labus J, et al. Patients with chronic visceral pain show sex-related alterations in intrinsic oscillations of the resting brain. J Neurosci 2013;33:11994-2002 CrossRef Medline

54. Amunts K, Kedo O, Kindler M, et al. Cytoarchitectonic mapping of the human amygdala, hippocampal region and entorhinal cortex: intersubject variability and probability maps. Anat Embryol (Berl) 2005;210:343-52 CrossRef Medline 